\title{
Pathologic reporting practices for breast cancer specimens after neoadjuvant chemotherapy-a survey of pathologists in academic institutions across the United States
}

\author{
Sonali Lanjewar ${ }^{1,2} \cdot$ Priyanka Patil $^{1} \cdot$ Susan Fineberg ${ }^{1}$
}

Received: 11 April 2019 / Revised: 21 June 2019 / Accepted: 22 June 2019 / Published online: 5 August 2019

(c) United States \& Canadian Academy of Pathology 2019

\begin{abstract}
Neoadjuvant chemotherapy is increasingly being used to treat primary invasive breast carcinoma. Response to neoadjuvant chemotherapy is an important determinant of prognosis. A multidisciplinary group published recommendations for standardization of pathologic reporting of postneoadjuvant chemotherapy specimens. Based on these recommendations, we sent a survey to 26 pathologists currently practicing breast pathology in academic centers across the United States. The survey consisted of six questions with yes/no answers. The pathologists were encouraged to add comments. We received responses from 23 breast pathologists from 19 centers. The questions and responses were as follows: 1 . Do you grade tumors after neoadjuvant chemotherapy?-17 (74\%) responded yes and $6(26 \%)$ responded no. 2. Do you routinely repeat hormone receptors, HER2/Neu results after neoadjuvant chemotherapy?-15 (65\%) responded yes and $8(35 \%)$ responded no. 3. If there are features of tumor regression/tumor bed at the margin but no actual tumor at the margin do you report this?-11 $(48 \%)$ responded yes and $8(35 \%)$ responded no and $4(17 \%)$ reported a variable practice. 4 . Do you report number of nodes with fibrosis/changes of regression?-17 (74\%) responded yes and $6(26 \%)$ responded no. 5. Do you report residual cancer burden score on your report or at least provide information on your report so clinicians can calculate residual cancer burden? $-17(74 \%)$ responded yes and $6(26 \%)$ responded no. 6. Do you have a specific synoptic for cases after neoadjuvant chemotherapy? - 5 (22\%) responded yes and $18(78 \%)$ responded no. The major reasons provided for nonadherence to recommended guidelines included pathologists were unaware of prognostic importance of providing the information, reporting practices were clinician driven and some pathologists were unaware of the recommendation. We document that academic breast pathology practices show significant variability in reporting of postneoadjuvant chemotherapy cases. We document barriers to standard practice and provide recommendations we hope will contribute to a more uniform reporting practice for these complex specimens.
\end{abstract}

\section{Introduction}

Neoadjuvant chemotherapy was first introduced in 1970 for management of inoperable breast cancer. In current times, neoadjuvant chemotherapy is gaining popularity as a treatment for early stage invasive breast cancer. Approximately $7-27 \%$ of women with new breast cancer are receiving

Susan Fineberg

sfineber@montefiore.org

1 Montefiore Medical Center and the Albert Einstein College of Medicine, Department of Pathology, Bronx, NY, USA

2 Methodist Lebonheur Hospital and University of Tennessee Health Science Center, Memphis, TN, USA neoadjuvant chemotherapy worldwide [1]. The increasing use of neoadjuvant chemotherapy stems from the fact that it can downstage both the tumor in the breast and axilla to allow for breast conserving surgery and improve quality of life by avoiding axillary dissection [2]. Response to neoadjuvant chemotherapy serves as an in vivo test of tumor chemosensitivity and provides important prognostic information, particularly with a pathological complete response, which is associated with a marked reduction in risk of distant relapse [3]. In addition, a pathological complete response after neoadjuvant chemotherapy is currently accepted by the US Food and Drug Administration as a path for accelerated drug approval [4].

Although distant recurrence rates and death from cancer are equally reduced by neoadjuvant chemotherapy and adjuvant chemotherapy, neoadjuvant chemotherapy carries 
the disadvantage of higher local recurrence rates when compared with adjuvant systemic therapy [5]. An important factor that can mitigate this risk is accurate pathologic assessment of residual disease after neoadjuvant chemotherapy. Response patterns of breast cancer after neoadjuvant chemotherapy are highly variable, however, and it is not uncommon to find widely scattered tumor foci or significant residual disease without obvious gross tumor mass. As a result, pathologic evaluation of these specimens is complex and requires both careful localization and appropriate sampling of the tumor bed, as well as reporting information which is not typically incorporated into non neoadjuvant chemotherapy pathology reports. Consistent and reproducible pathologic assessment of response to neoadjuvant chemotherapy is hindered by a lack of universal reporting protocols.

In 2015, an international multidisciplinary working group convened by the Breast International Group-North American Breast Cancer Group (BIG-NABCG) published recommendations for the standardization of pathologic evaluation and reporting of postneoadjuvant breast cancer specimens in clinical trials [6]. These recommendations provide a standardized approach to evaluate postneoadjuvant chemotherapy breast cancer specimens whether part of a clinical trial or not. Variability in pathology reporting among pathologists could contribute to inaccurate evaluation of the postneoadjuvant chemotherapy pathology specimen and result in suboptimal surgical and oncologic management of patients. Our goal was to assess variability in the pathology reporting practices of postneoadjuvant chemotherapy specimens by breast pathologists in academic pathology departments in the United States and to better understand barriers to standardized reporting.

\section{Materials and methods}

We sent a questionnaire to 26 pathologists in academic pathology departments who were practicing breast pathology across the United States in 2017 and 2018. The questions were based on pathology reporting recommendations for postneoadjuvant breast cancer specimens as outlined in the publication by the international working group convened by the BIG-NABCG [6]. It consisted of six questions with simple yes or no answers. The pathologists were encouraged to add any comments in the survey. The survey questions were as follows:

1. Do you grade tumors after neoadjuvant chemotherapy?

2. Do you routinely repeat hormone receptors HER2/ Neu results after neoadjuvant chemotherapy?

3. If there are features of tumor regression/tumor bed at the margin but no actual tumor at the margin do you report this?

4. Do you report number of nodes with fibrosis/changes of regression?

5. Do you report residual cancer burden score on your report or at least provide information on your report so clinicians can calculate residual cancer burden score?

6. Do you have a specific synoptic for cases after neoadjuvant chemotherapy?

Any other comments/thoughts appreciated.

\section{Results}

We received a response from 23 of the 26 breast pathologists from 19 academic pathology departments. The 19 academic centers included:

Montefiore Medical Center/Albert Einstein school of Medicine-Bronx, New York.

Memorial Sloan Kettering Cancer Center-New York, New York.

Columbia University Medical Center-New York, New York.

Weill Cornell Medical Center-New York, New York.

Mount Sinai Medical Center-New York, New York.

SUNY Downstate Medical Center-Brooklyn, New York.

New York University-New York, New York

Stanford University Medical Center-Stanford, California.

Yale University Hospital-New Haven, Connecticut.

Brigham and Women's Hospital and Dana-Farber Cancer Institute-Boston, Massachusetts.

Brown University-Providence, Rhode Island.

Emory University-Atlanta, Georgia.

Indiana University-Indianapolis, Indiana.

University of Florida-Gainesville, Florida.

Medical College of Wisconsin-Milwaukee, Wisconsin.

University of Pittsburgh Medical Center-Pittsburgh, Pennsylvania.

Henry Ford Hospital-Detroit, Michigan.

East Carolina University-Greenville, North Carolina.

MD Anderson Cancer Center-Houston, Texas.

A summary of the responses is presented in Table 1. Note that in four instances two pathologists practiced in the same institution, and in three of these four all responses were concordant. For one institution two pathologists reported a different practice regarding reporting tumor regression/tumor bed at the margin, however responses to the other five questions were concordant. Results for each question as well as representative specific comments from the participants are detailed below. 
Table 1 Summary of responses from 23 pathologists to postneoadjuvant chemotherapy breast cancer pathology reporting questionnaires

\begin{tabular}{|c|c|c|}
\hline Questionnaires & Yes & $\overline{\mathrm{No}}$ \\
\hline Do you grade tumors after neoadjuvant chemotherapy? & 17 & \\
\hline Do you routinely repeat hormone receptors HER $2 / \mathrm{Neu}$ results after neoadjuvant chemotherapy? & 15 & 8 \\
\hline $\begin{array}{l}\text { If there are features of tumor regression/tumor bed at the margin but no actual tumor at the } \\
\text { margin do you report this? }\end{array}$ & 11 & 8 \\
\hline Do you report number of nodes with fibrosis/changes of regression? & 17 & 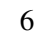 \\
\hline $\begin{array}{l}\text { Do you report residual cancer burden score on your report or at least provide information on } \\
\text { your report so clinicians can calculate residual cancer burden? }\end{array}$ & 17 & \\
\hline Do you have a specific synoptic for cases after neoadjuvant chemotherapy? & 5 & 18 \\
\hline
\end{tabular}

${ }^{a}$ Four pathologists reported a variable practice

\section{Do you grade tumors after neoadjuvant chemotherapy?}

Seventeen of the twenty-three pathologists (74\%) responded yes this question and six (26\%) responded no.

Four of the six pathologists who reported they do not grade tumors after neoadjuvant chemotherapy made the following comments:

"Not typically. I will re-grade postneoadjuvant chemotherapy if minimal to no chemoresponse and clearly different from the previous biopsy"

"Usually no! In our experience, the mitotic rate drops dramatically, potentially turning a poorly differentiated tumor into a well to moderately differentiated, a conversion that doesn't make a whole lot of sense."

"No. We report the pretreatment grade."

"No. There is no evidence showing correlation of tumor grade with prognosis after neoadjuvant chemotherapy."

Five of the seventeen pathologists who reported that they do grade tumors after neoadjuvant chemotherapy made the following comments:

"Yes;we suggest making a comment if obvious treatment-related cytologic changes are seen in malignant cells and the atypia is significant enough to cause a grade shift."

"Yes, except for cases in which the tumor cells show prominent treatment effect; in those cases, we say that accurate grading is precluded by prominent treatment effect."

"Typically yes if there is enough tumor. I find it helpful to compare with the core if it's available. If there are only scattered cells or the invasive carcinoma looks different from the core (treatment effect, bizarre nuclei, etc.) I just say- residual carcinoma with treatment effect."

"We do grade them and they usually stay the same grade but are less cellular. The rare cases that have a different grade are of interest - but I haven't seen any studies showing what this signifies clinically. It would be of interest to note whenever there is a marked difference in grade"

"Yes. The majority of carcinomas have been grade 3 on biopsy"

\section{Do you routinely repeat hormone receptors HER2/ Neu results after neoadjuvant chemotherapy?}

Fifteen of the twenty-three pathologists (65\%) responded yes to this question and eight (35\%) responded no.

Five of the eight pathologists who report they do not routinely repeat hormone receptors HER2/Neu results after neoadjuvant chemotherapy made the following comments:

"No unless as per College of American Pathologists/ American Society of Clinical Oncology guideline recommendations (e.g. Repeat HER-2 in grade 3, previous HER-2 negative)"

"For grade 3 (grade 3 on biopsy) tumors that are HER2 negative on biopsy we repeat HER2. Other markers are not repeated."

"We do not routinely repeat receptors after neoadjuvant systemic therapy decisions have already been made at this point. We assess each case individually. We do repeat on cases with substantial residual tumor that were previously negative and morphology suggesting that the tumor could be positive. On prior triple negative tumor with classic morphology, repeating the receptors and finding focal estrogen receptor positivity can make some patients ineligible for triple negative tumor clinical trials."

"We do not routinely repeat markers after neoadjuvant chemotherapy (but we sometimes do them if we never actually reviewed the outside markers or performed them at out hospital before treatment)"

"I typically repeat them, particularly if the patient has never had testing performed at our hospital. If the tumor is HER2 + and estrogen receptor positive on the core I don't repeat."

Three of the fifteen pathologists who report they do routinely repeat hormone receptors HER2/Neu results after neoadjuvant chemotherapy made the following comments:

"Yes- this is per clinical request to do so"

"Yes, our oncologists have been requesting this"

"We do repeat all three again, because patients are on protocols and this information may be of value in the future.

Estrogen receptor should be repeated on cancers that were estrogen receptor negative prior to treatment because 
there are at least 2 studies showing that patients with cancers that are found to be Estrogen receptor positive after treatment have a better prognosis and should be treated with endocrine therapy.

The likelihood of a cancer converting to being HER2 positive after treatment is very low $(<5 \%)$ and has an uncertain clinical significance since the patient also has HER2 negative cancer. We recently had one of these rare cases and she did get HER2 targeted therapy.

Repeating positive results has little clinical value (except that loss of estrogen receptor and/or HER2 is a poor prognostic factor) as negative results generally don't change treatment.

Changes in progesterone receptor likely have little importance.

So - definitely estrogen receptor on previously negative cancers but repeating the others may be optional. It would be helpful for your group to find out what the medical oncologists would find useful."

\section{If there are features of tumor regression/tumor bed at the margin but no actual tumor at the margin do you report this?}

Eleven of the twenty-three pathologists (48\%) responded yes to this question, eight $(35 \%)$ responded no, and four (17\%) reported a variable practice. Notably, for two pathologists from the same institution, one responded yes and the other responded no to this question.

Four of the eight pathologists who responded that they do not report features of tumor regression/tumor bed at the margin made the following comments:

"We generally do not mention whether the tumor bed extends to the margin (although we often indicate this at the time of gross intraoperative assessment and sometimes do a frozen section)"

"I don't. Not sure if there is a recommendation on this. If there are tumor bed changes in separately submitted margins in the lumpectomy cases I will mention it, but don't say whether these changes extend to a final margin."

"We do not report tumor bed at the margin for 2 reasons. First, there are no data indicating that this is of prognostic significance with regard to risk of local recurrence. Second, given that our surgeons DO NOT attempt to excise the full extent of the original tumor, every case should theoretically have tumor bed at the margin."

"No. But if biopsy site change is truncated at the margin, I report it."

One of the 11 pathologists who responded that they do report features of tumor regression/tumor bed at the margin made the following comment:

"We do report obvious tumor bed at the margin. If the patient has had a pathologic complete response, this would not be an indication for more surgery. If there is scattered tumor throughout the tumor bed, then it is helpful for the clinicians to know that this "negative" margin is not very reliable in predicting if there is cancer left in the breast. Whether or not to do more surgery in this setting is controversial. This could be useful information in the future when looking at factors associated with local recurrence."

The four pathologists who responded that they variably report tumor regression/tumor bed at the margin made the following comments:

"Variable. We don't have policy for reporting this"

"Yes, sometimes- only if there is residual tumor consisting of small cells and nests widely scattered across the fibrotic tumor bed."

"Have done this a few times, not sure if I should continue."

"May make a comment but as long as there are no tumor cells we call the margin negative."

\section{Do you report number of nodes with fibrosis/ changes of regression?}

Seventeen of the twenty-three pathologists (74\%) responded yes to this question and six $(26 \%)$ reported no.

Four of the six pathologists who indicated that they do not report the number of nodes with fibrosis/changes of regression made the following comments:

"Just include a general statement about all lymph nodes whether there is evidence of fibrosis"

"We do not report the number of nodes with treatment effect (although we sometimes mention in a general way that some nodes have fibrosis consistent with treatment effect)"

"No, but this is a good idea, and so we just added it to our synoptic report"

"No. But I will report biopsy site changes in the lymph nodes. Our physicians want to know that."

Three of the 17 pathologists who indicated that they do report the number of nodes with fibrosis/changes of regression made the following comments:

"Yes- We report the number of nodes with treatment effect. Even when we say "no definite" our radiologyoncology folks often ask us to re-review to see if there was any evidence of prior involvement. We can really only be sure when there is good scar there, so I only report if I see that."

"Lymph nodes with fibrosis and changes like macrophages are reported as an indication of a previously positive node. We also go to great details about the clip and associated biopsy site changes. We also image the node if we know that a clip has been previously been left after core biopsy." 
"We do report the number of nodes that look like they were previously involved by carcinoma. If there was a needle biopsy that showed cancer, this is a reassuring finding that the involved node was identified and removed - and it is of more concern if all the nodes look normal. It is also helpful to know what the likely tumor burden was before treatment. However, it is also helpful for the clinicians to know that metastases can regress and not leave any histological evidence. They do not place clips in biopsied nodes prior to treatment - although I think this is a good idea."

\section{Do you report residual cancer burden score on your report or at least provide information on your report so clinicians can calculate residual cancer burden score?}

Seventeen of the twenty-three pathologists (74\%) responded yes to this question and six $(26 \%)$ reported no.

Three of the six pathologists who indicated that they do not report residual cancer burden score and do not provide information so clinicians can calculate residual cancer burden score made the following comments:

"No, but certain individual pathologists, myself included, report the cellularity in addition to size. Our clinicians don't use residual cancer burden score as a guide."

"Never used it"

"PA/residents do the gross and cannot rely on the documentation of gross to provide specifics for calculation of residual tumor."

Three of the seventeen pathologists who indicated that they do report residual cancer burden score or atleast provide information so clinicians can calculate residual cancer burden score made the following comments:

"We have a template that includes the information necessary for calculating the residual cancer burden score. The template also includes residual cancer burden score index and RCB class, with an indication that this is optional. I always go ahead and include it."

"This was a recent topic of tumor board discussions because we report: 1. 'Span of residual invasion' as a surrogate for 'Tumor bed' since it seemed that is how it would be defined but there have been cases where there was debate if the span of residual DCIS should be included in this measurement. Their instruction just state 'span of residual cancer' is to be used as the 'tumor bed' by their definition. I wanted to avoid using 'tumor bed' in our reports because it is so often confused with scar/treatment changes rather than residual cancer. 2. Cellularity due to invasion (in the area spanning the invasion). Again, we are using different terms than in the calculator to avoid confusion since as I understand it the 'overall cancer cellularity' then subtracts out the 'percent that is in situ' in the residual cancer burden score calculator. It would be helpful to have clarity from the residual cancer burden score folks on this. I think there is a lot of variability on how this is done."

"I often find it difficult to estimate cellularity. I use the residual cancer burden score cellularity guide provided on the MD Anderson Cancer Center website"

\section{Do you have a specific synoptic for cases after neoadjuvant chemotherapy?}

Five of the twenty-three pathologists (22\%) answered yes to this question and eighteen (78\%) responded no.

\section{Discussion}

Neoadjuvant chemotherapy is increasingly being used to treat localized invasive breast cancer. Pathologic assessment of response to neoadjuvant chemotherapy provides important prognostic information. In patients who do not achieve a pathological complete response accurate quantification of residual disease is critical for further management and directs need for additional or alternate therapies or additional surgery [7-9]. An international multidisciplinary working group convened by the Breast International GroupNorth American Breast Cancer Group (BIG-NABCG) published recommendations for the standardized pathologic reporting of postneoadjuvant breast cancer specimens [6]. Based on these recommendations, we sent a questionnaire to 23 pathologists from 19 academic institutions practicing breast pathology across the United States. We found significant variability in adherence to these reporting guidelines. The major reasons provided for nonadherence to recommended guidelines included pathologists reported they were unaware of prognostic importance or clinical value of providing the information, reporting practices were clinician driven and in some cases the pathologists were unaware of the recommendation.

Both the College of American Pathologists and the international working group recommend grading of residual carcinoma after neoadjuvant chemotherapy using the Nottingham combined histologic grade $[6,10]$. In our survey, 6 of 23 pathologists reported that they do not grade tumors after neoadjuvant chemotherapy and one reason provided was that the tumor grade after neoadjuvant chemotherapy was not linked to prognosis. The most widely used pathologic classification systems to evaluate tumor response to neoadjuvant chemotherapy are the AJCC yp stage and the residual cancer burden score, neither of which include posttreatment tumor grade $[11,12]$. However, recent publications have demonstrated that the addition of post-treatment grade to residual cancer burden score improves prediction of long-term outcome over residual cancer burden score 
alone [13]. Grade shift owing to treatment-related cytologic atypia can be seen in up to $32 \%$ of cases and has not been linked to prognosis [14, 15]; this can be addressed by comparison to pretherapy material. However, low mitotic rate score in residual tumor after neoadjuvant chemotherapy has been associated with improved disease-free survival $[16,17]$. Moreover, post therapy Ki67 provided more prognostic information than pretherapy levels as well as change in Ki67 from pre to post therapy [18]. Information on post therapy proliferative activity, which is provided by the mitotic rate score component of the Nottingham histologic grade, is prognostic and should be reported. We suggest providing both pre and post therapy tumor grade in pathology reports of post neoadjuvant chemotherapy breast cancer specimens. This would allow for assessment of change, if any, of the critical components of overall grade.

After neoadjuvant chemotherapy, documentation of treatment effect in both positive and negative lymph nodes is a required element in the College of American Pathologists protocol for the examination of specimens from patients with invasive carcinoma of the breast [10]. Even in patients with nodal pathological complete response, local recurrence rates are higher in those who had positive nodes prior to therapy, hence documentation of treatment effect in lymph nodes supports pretherapy nodal involvement and provides important prognostic information [19]. If lymph node involvement was documented by biopsy prior to therapy, presence of treatment effect in negative nodes indicates that the involved node was removed. Although concerns that previously positive nodes can appear normal after therapy have been raised, this was noted in only $3 \%$ of patients with complete axillary dissection [20]. In addition to reporting treatment effect in nodes, the international working group also recommends that the number of nodes with treatment effect be included in the pathology report. Recent National Comprehensive Cancer Network guidelines state that the need for adjuvant radiation therapy is based on the maximal disease from pretherapy tumor [21]. Reporting the number of nodes with treatment effect therefore provides information on pretherapy nodal disease burden and may influence the need for post therapy radiation therapy, particularly after mastectomy. In our survey, $26 \%$ of pathologists reported that they do not include number of nodes with treatment effect in postneoadjuvant chemotherapy specimens. Evidence suggests this information provides important prognostic information and can be easily included in pathology reports. Inclusion of this element within College of American Pathologists protocols might increase adherence to this guideline.

In our survey, $26 \%$ of pathologists indicated that they do not provide information on pathology reports which would allow for calculation of the residual cancer burden score. Both the AJCC and the international working group recommend use of both the residual cancer burden score and ypTNM for pathologic quantification of residual disease after neoadjuvant chemotherapy $[6,11]$. The ypTNM stage uses the largest contiguous focus of residual invasive carcinoma in the breast and nodes to determine ypTN stage, whereas residual cancer burden score additionally incorporates information on tumor bed size, cancer cellularity and percentage of in situ carcinoma that is subtracted from the total cellularity [12]. Residual cancer burden score can be calculated using an online calculation tool at the website www.mdanderson.org/breastcancer.RCB. Residual cancer burden score divides patients into four risk groups which stratify for distant relapse [12]. This stratification tool provides more prognostic information than AJCC ypTN staging and has shown to be both reproducible amongst pathologists, and prognostic across all breast cancer subtypes [22-25]. Accurate calculation of residual cancer burden score requires careful mapping and extensive sampling of the gross specimen, particularly when residual tumor is less well defined, and one pathologist in our survey commented that she did not consider grossing done by her pathology assistants or residents to be reliable enough for calculating residual cancer burden score. One pathologist noted that clinicians did not use residual cancer burden score as a guide. Among pathologists who do report residual cancer burden score it was noted that some of the guidelines for determining key elements of residual cancer burden score require further clarification. It appears that barriers to both use and accuracy of residual cancer burden score include complexity of specimen handling as well as lack of clarity of guidelines for use. Although a series of instructions are present on the residual cancer burden score website as well as video tutorial, a forum for direct questions about residual cancer burden score application might be helpful. In addition, incorporation of key elements for residual cancer burden score calculation into College of American Pathologists cancer reporting synoptics along with instructions would assist pathologists in reporting residual cancer burden score. A recent publication addresses some of the pitfalls and less common situations encountered when calculating residual cancer burden score [26].

The international working group recommends documenting tumor bed/tumor regression extending to margins, however only 11 of 23 pathologists in our study report this. Explanations provided for nonreporting include absence of demonstrated prognostic significance, whereas some pathologists noted that they were unaware of this guideline. A common pattern of residual tumor after neoadjuvant chemotherapy consists of multiple small tumor foci scattered throughout a tumor bed, and the working group notes that in these cases carcinoma may extend beyond a histologically negative margin. A recent meta-analysis that included 4756 women from 10 randomized trials reported 
that neoadjuvant chemotherapy was associated with higher local recurrence rates than was adjuvant chemotherapy: the 15 year local recurrence was $21.4 \%$ for neoadjuvant vs $15.9 \%$ for adjuvant chemotherapy [5]. In patients who received neoadjuvant chemotherapy, non-mass enhancement on magnetic resonance imaging, which is a pattern suggesting microscopic tumor foci, was associated with higher local recurrence rates [27]. Two participants in our survey highlighted the importance of reporting tumor bed at the margin if tumor bed contained scattered tumor foci, and evidence suggest this may represent an informed approach. One pathologist noted that "surgeons do not attempt to excise the full extent of the original tumor." This indicates tumor bed at the margin is not uncommon and may not be an indication that re-excision is necessary. However, we suggest that this information combined with imaging findings as well as a general description of residual tumor distribution might inform further management in select cases. Finally, as noted by a participant in our survey, information on tumor bed at the margin "could be useful information in the future when looking at factors associated with local recurrence."

Change in biomarker expression after neoadjuvant chemotherapy has been reported with a mean prevalence of up to $18 \%$ for estrogen receptor, $32 \%$ for progesterone receptor, and $6 \%$ for HER2/Neu [28, 29]. There is currently no consensus guideline for retesting of these biomarkers after neoadjuvant chemotherapy and practices vary widely. In our survey, only $65 \%$ of pathologists report routine retesting for estrogen and progesterone receptors and HER2/Neu after neoadjuvant chemotherapy, reflecting a highly variable practice in academic pathology practice across the United States. Furthermore, some of the pathologists who report routine retesting noted this was driven by clinical request from oncologists and/or required as part of clinical protocols. The College of American Pathologists recommends that if biomarkers were negative prior to therapy that retesting should be performed on residual invasive carcinoma after neoadjuvant chemotherapy. In contrast, the international working group only recommends consideration for retesting in this setting $[6,10]$. Expression of one of these biomarkers after therapy in a previously triple-negative carcinoma could result in switch to targeted therapy and conversion from hormone receptor negative to positive is associated with improved survival [30, 31]. Conversely loss of hormone receptors or HER2/Neu have been associated with worse outcome, however this change is unlikely to inform therapy and the international working group does not recommend routine reassessment for tumors which are hormone receptor and HER2/Neu positive pretherapy. This situation is not specifically addressed in College of American Pathologists cancer protocols
$[6,10,30,32]$. Tumor heterogeneity, changes induced by therapy, technical factors and clinical protocols are just a few of the factors that contribute to both biomarker switch after neoadjuvant chemotherapy and decisions related to retesting practices. This is a complex issue and should be addressed more specifically within the College of American Pathologists cancer biomarker protocols, possibly in the form of an algorithmic approach.

Neoadjuvant chemotherapy has evolved from a treatment used for patients with inoperable invasive breast cancer to one which now can be considered in all patients eligible for adjuvant therapy [33]. Accurate quantification and qualification of residual disease is complex and the international working group recommends reporting of additional elements currently not incorporated into College of American Pathologists cancer protocols. It is critically important that pathologists provide accurate and reproducible reports for optimal management of breast cancer patients after neoadjuvant chemotherapy. In our survey, we have demonstrated that even among academic breast pathologists there is significant variability in reporting practices with variable adherence to the international working group guidelines. In our paper, we highlight some of the international working group reporting recommendations, provide evidence to support the clinical importance of reporting these elements, and provide suggestions to allow for incorporation of some of these elements into pathology reports from breast cancer specimens resected after neoadjuvant chemotherapy. Creation of universal College of American Pathologists protocols for reporting postneoadjuvant chemotherapy breast cancer specimens, created in collaboration with a multidisciplinary team of physicians involved in breast cancer treatment, would help standardize reporting practices among pathologists and ensure that significant variables required for optimum patient care are reported.

Acknowledgements We acknowledge the pathologists who contributed to this study with their responses to our survey and their comments provided with study questionnaire: Dr. Bryan Harmon, Dr Farbod Darvishian, Dr Michael Gilcrease, Dr. Kimberly Allison, Dr. Hanina Hibshoosh, Dr. Malini Harigopal, Dr. Diane Hamele-Bena, Dr. Ruth Asirvatham, Dr. Julie Jornes, Dr. Bill Li, Dr. Rohit Bhargava, Dr. Raavi Gupta, Dr Shabnam Jaffer, Dr. Paula Ginter, Dr. Kimberly Cole, Dr Sunil Badve, Dr. Susan Lester, Dr. Syed Hoda, Dr. Timothy D'Alfonso. Dr. Yihong Wang, Dr. Fatima Zahra Aly, Dr Stuart Schnitt, Dr Susan Fineberg. We acknowledge editorial assistance provided by Jenna Ansell, and Matthew Shear.

\section{Compliance with ethical standards}

Conflict of interest The authors declare that they have no conflict of interest.

Publisher's note: Springer Nature remains neutral with regard to jurisdictional claims in published maps and institutional affiliations. 


\section{References}

1. Vaidya JS, Massarut S, Vaidya HJ, Alexander EC, Richards T, Caris JA, et al. Rethinking neoadjuvant chemotherapy for breast cancer. Brit Med J. 2018;360:j5913.

2. Hayes DF, Schott AF. Neoadjuvant chemotherapy: What are the benefits for the patient and investigator? JNCI Monogr 2015;51:36-9.

3. Cortazar P, Zhang L, Untch M, Mehta K, Costantino JP, Wolmark $\mathrm{N}$, et al. Pathological complete response and long-term clinical benefit in breast cancer: the CTNeoBC pooled analysis. Lancet. 2014;384:164-72.

4. Prowell TM, Pazdur R. Pathological complete response and accelerated drug approval in early breast cancer. N Engl J Med. 2012;366:2438-41.

5. Early Breast Cancer Trialists' Collaborative Group (EBCTG). Long- term outcomes or neoadjuvant versus adjuvant chemotherapy in early breast cancer: meta-analysis of individual patient data from ten randomized trials. Lancet Oncol. 2018;19:27-39.

6. Provenzano E, Bossuyt V, Viale G, Cameron D, Badve S, Denkert $\mathrm{C}$, et al. Standardization of pathologic evaluation and reporting of postneoadjuvant specimens in clinical trials of breast cancer: recommendations from an international working group. Mod Pathol 2015;28:1185-201.

7. Fisher B, Bryant J, Wolmark N, Mamounas E, Brown A, Fisher ER, et al. Effect of preoperative chemotherapy on the outcome of women with operable breast cancer. J Clin Oncol. 1998;16:2672-85.

8. Masuda N, Lee S-J, Ohtani S, Im YH, Lee ES, Yokota I, et al. Adjuvant capecitabine for breast cancer after preoperative chemotherapy. N Engl J Med. 2017;376:2147-59.

9. Von Minckwitz G, Huang C, Mano MS, Loibl S, Mamounas EP, Untch M, et al. Trastuzamab Emtansine for Residual Invasive HER2-positive breast cancer. N Engl J Med. 2019;380:617-28.

10. Patrick L, Fitzgibbons P, Bose S, Chen Y, Baca ME, Edgerton M et al. Protocol for the examination of specimens from patients with invasive carcinoma of the breast. http://www.cap.org/ca cnerprotocols. 2018.

11. Gabriel N, Hortobagyi, Connely J, D’Orsi CJ, Edge S, Mittendorf E, et al. AJCCCancer Staging Manual AJCC Cancer Staging Manual. 8th edn. New York, NY:Springer 2017.

12. Symmans WF, Petintinger F, Hatzis C, Rajan R, Kuerer HM, Valero V, et al. Measurement of residual breast cancer burden to predict survival after neoadjuvant chemotherapy. J Clin Oncol. 2007;25:4414-22.

13. Sheri A, Smith IE, Johnston S, A'Hern R, Nerurkar A, Jones RL, et al. Residual proliferative cancer burden to predict long-term outcome following neoadjuvant chemotherapy. Ann Oncol 2015;26:75-80.

14. Sharkey FE, Addington SL, Fowler LJ, Page CP, Cruz AB. Effects of preoperative chemotherapy on the morphology of resectable breast carcinoma. Mod Pathol. 1996;9:893-900.

15. Sahoo SC, Lester SC. Pathology of breast carcinomas after neoadjuvant chemotherapy: an overview with recommendations on specimen processing and reporting. Arch Pathol Lab Med. 2009;133:633-42.

16. Penault-Llorca F, Raoelfils AC, Cayre C, Mouret-Reynier MA, Mishellany F, Gimbergues $\mathrm{P}$, et al. Changes and predictive and prognostic value of the miotic index, KI67, cyclin D1 and cyclooxegenase 2 in 710 operable breast cancer patients treated with neoadjuvant chemotherapy. Oncologist 2008;13:1235-45.

17. Diaz J, Stead L, Shapiro N, Newell R, Loudig O, Lo Y, et al. Mitotic counts in breast cancer after neoadjuvant systemic therapy and development of metastatic disease. Breast Cancer Res Treat 2013;138:91-7.

18. Von Minckowitz G, Schmitt WD, Loibi S, Muller BM, Biohmoer JU, Sinn BV, et al. Ki67 measured after neoadjuvant chemotherapy for primary breast cancer. Clin Cancer Res 2013;19:4521-31.

19. Mamouonas EP, Anderson S, DIgman JET, Bear H, Julian T, Geyer C, et al. Predictors of locoregional recurrence after neoadjuvant chemotherapy: results from combined analysis of national surgical adjuvant breast and bowel project B-18 and B26. J Clin Oncol. 2012;30:3960-6.

20. Barrio AV, Mamtanio A, Edelweiss M, Eaton A, Stempel M, Murray MP, et al. How often is treatment effect identified in axillary nodes with a pathologic complete response after neoadjuvant chemotherapy. Ann Surg Oncol. 2016;23:3475-80.

21. Gradishar W, Anderson B, Abraham J, Aft R, Agnese D, Allison $\mathrm{K}$, et al. NCCN Clinical practice guidelines in oncology Breast Cancer Version 3. 2018. www.nccn.org/professionals/physicisn.

22. Symmans W, Caimiao W, Rebekah G, Yu X, Zhang Y, Liu M, et al. Long term prognostic risk after neoadjuvant chemotherapy associated with residual cancer burden and breast cancer subtype. J Clin Oncol 2017;35:1049-60.

23. Thomas J, Provenzano, Hiller 1, Dunn J, Blenkinsop C, Grybowicz L, et al. Central pathology review with two-stage quality assurance for pathologic response after neoadjuvant chemotherapy in the ARTemis Trial. Mod Pathol. 2017;30:1069-77.

24. Lee HJ, Park IA, Song IH, Kim S, Jung KH, Ahn J, et al. Comparison of pathologic response evaluation systems after anthracycline with/without taxane -based neoadjuvant chemotherapy among different subtypes of breast cancer. PLoS ONE. 2015;10:e0137885.

25. Naidoo K, Parham D, Pinder SE. An audit of residual cancer burden reproducibility in a UK context. Histopathology. 2017;70:2170-222.

26. Bossuyt V. Processing and reporting of breast specimens in the neoadjuvant setting. Surg Pathol Clin. 2018;11:213-30.

27. Shin S, Cho N, Lee H, Kim S, Yi A, Kim S, et al. Neoadjuvant chemotherapy and surgery for breast cancer:Preoperative MRI features associated with local recurrence. Radiol. 2018;289:30-38.

28. Jabbout M, Massad C, Boulos F. Variability in hormone and growth factor receptor expression in primary versus recurrent, metastatic and post-neoadjuvant breast carcinoma. Breast Cancer Res Treat. 2012;135:29-37.

29. Zhang N, Moran M, Huo Q, HAffty B, Yang Q. The hormonal receptor status in breast cancer can be altered by neoadjuvant chemotherapy: a meta-analysis. Cancer Invest. 2011;29:594-8.

30. Lim SK, Lee MH, Park HI, You JY, Nam B, Kim BN, et al. Impact of molecular subtype conversion of breast cancers after neoadjuvant chemotherapy on clinical outcome. Cancer Res Treat. 2016;48:133-41.

31. Tacca O, Penault-Llorca F, Abrial C, Mouret-Reynier MA, Raoelfis I, Durando X, et al. Changes in and prognostic value of hormone receptor status in a series of operable breast cancer patient treated with neoadjuvant chemotherapy. Oncologist. 2007;12:636-43.

32. Guarneri V, Dieci MV, Barbieri E, Placentini F, Omarini C, Ficarra G, et al. Loss of HER2 positivity and prognosis after neoadjuvant therapy in HER2-positive breast cancer patients. Ann Oncol 2013;24:2990-4.

33. Amoroso V, Generali D, Buchholz T, Cristofanilli M, Pedersini R, Curigilano $\mathrm{G}$, et al. International expert consensus on primary systemic therapy in the management of early breast cancer: Highlights of the fifth symposium on primary systemic therapy in the management of operable breast cancer, Cremona, Italy (2013). JNCI Monogr. 2015;2015:90-6. 